\title{
Polarisation-independent sub-picosecond flat-top pulse generation for ultra-fast 640 Gbit/s gating
}

Oxenløwe, Leif Katsuo; Galili, Michael; Mulvad, Hans Christian Hansen; Slavik, Radan; Clausen, Anders; Jeppesen, Palle

Published in:

Proceedings, OFC

Publication date:

2009

Document Version

Publisher's PDF, also known as Version of record

Link back to DTU Orbit

Citation (APA):

Oxenløwe, L. K., Galili, M., Mulvad, H. C. H., Slavik, R., Clausen, A., \& Jeppesen, P. (2009). Polarisationindependent sub-picosecond flat-top pulse generation for ultra-fast $640 \mathrm{Gbit} / \mathrm{s}$ gating. In Proceedings, OFC (pp. 1-3). IEEE.

\section{General rights}

Copyright and moral rights for the publications made accessible in the public portal are retained by the authors and/or other copyright owners and it is a condition of accessing publications that users recognise and abide by the legal requirements associated with these rights.

- Users may download and print one copy of any publication from the public portal for the purpose of private study or research.

- You may not further distribute the material or use it for any profit-making activity or commercial gain

- You may freely distribute the URL identifying the publication in the public portal 


\title{
Polarisation-independent sub-picosecond flat-top pulse generation for ultra-fast $640 \mathrm{Gbit} / \mathrm{s}$ gating
}

\author{
L.K. Oxenløwe (1), M. Galili (1), H.C.H. Mulvad (1), R. Slavík (2), A.T. Clausen (1) and P. Jeppesen (1) \\ 1: DTU Fotonik, Technical University of Denmark, Building 343, DK-2800 Kgs. Lyngby,Denmark, lkox@fotonik.dtu.dk \\ 2: Institute of Photonics and Electronics, AS CR, Chaberská 57, 18251 Prague, Czech Republic
}

\begin{abstract}
An 840 fs wide flat-top pulse is generated in a polarisation-independent optical differentiation scheme and used for $640 \mathrm{Gbit} / \mathrm{s}$ demultiplexing. The timing jitter tolerance is improved by a factor of three to $310 \mathrm{fs}$.
\end{abstract}

(C) 2007 Optical Society of America

OCIS codes: (190.4360)Nonlinear optics, devices; (060.2330) Fiber optics communications

\section{Introduction}

The demand for higher telecom bandwidth is steadily increasing. One way to increase the channel capacity is to increase the serial data rate as in e.g. [1]. At high bit rates, it becomes increasingly challenging to find pulse sources with sufficiently low timing jitter. Therefore it can be very advantageous to have a switch with a high tolerance to timing jitter, e.g. by the use of flat-top (FT) control pulses in an ultra-fast switch. For $160 \mathrm{Gbit} / \mathrm{s}$, various approaches have been reported [2-4]. Beyond $160 \mathrm{Gbit} / \mathrm{s}$, a promising scheme is based on optical differentiation in a long-period fibre grating (LPG) [5]. This scheme has the great advantage of being able to handle arbitrary pulse durations. We recently reported on demonstrations at $640 \mathrm{Gbit} / \mathrm{s}$ [6], which were successful, yet marginally so. The reasons for this are that the bandwidth of the then used LPG was only about $6 \mathrm{~nm}$ resulting in wider than optimum pulses ( $~ 1.4 \mathrm{ps}$ FWHM with some residual pulse tails) yielding $>3 \mathrm{~dB}$ penalty relative to the $10 \mathrm{Gbit} / \mathrm{s}$ back-to-back, and that the LPG was polarisation dependent.

In this paper, we report on a significant breakthrough in the performance of our switch by significant modification of the LPG fabrication technique and more careful design of the flat-top pulse shaper for operation at $640 \mathrm{Gbit} / \mathrm{s}$. Doing so, we were able to generate sub-picosecond (FWHM) flat-top pulses in this simple all-fiber scheme, irrespective of the input polarisation. Specifically, we create an $840 \mathrm{fs}$ FWHM flat-top pulse, and show for the first time the use of a sub-picosecond flat-top pulse in a systems experiment. The pulse not only allows for error-free performance with less than $0.5 \mathrm{~dB}$ penalty to the $10 \mathrm{Gbit} / \mathrm{s} \mathrm{b}-\mathrm{b}$, but also yields jitter tolerant operation at $640 \mathrm{Gbit} / \mathrm{s}$. We find a 310 fs timing tolerance, corresponding to $20 \%$ of the bit duration, which is almost a factor three more than for a gaussian shaped pulse with the same 1/e-width.

\section{Experimental procedure}

The experimental set-up used is sketched in Fig. 1. The data signal is generated by an erbium glass mode-locked

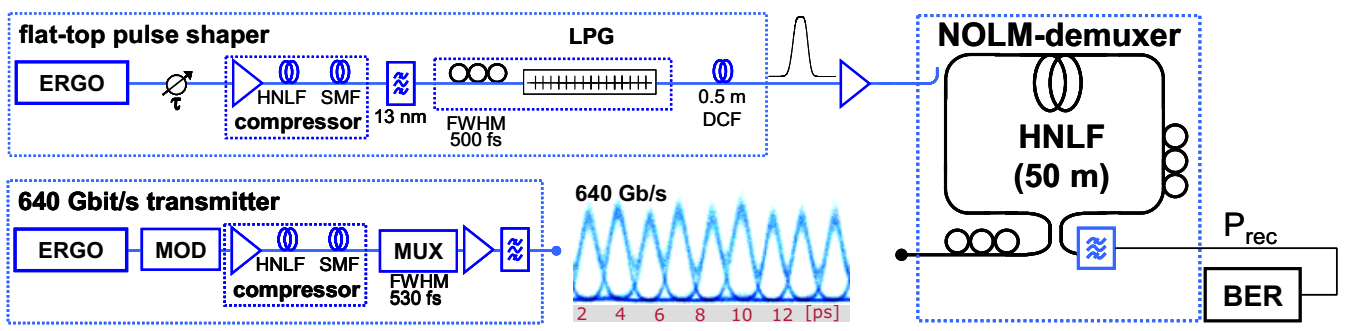

Figure 1. Setup for shaping of 840 fs flat-top pulse for $640 \mathrm{Gbit} / \mathrm{s}$ switching.

laser (ERGO) at $10 \mathrm{GHz}$ and $1557 \mathrm{~nm}$. The pulses are data modulated with a $2^{7}-1$ PRBS (MOD). The data pulses are then chirped by Self Phase Modulation (SPM) in $400 \mathrm{~m}$ of dispersion flattened highly non-linear fiber (HNLF). The positive dispersion in the remainder of the transmitter, corresponding to $20 \mathrm{~m} \mathrm{SMF}$, linearly compresses the data pulses to $\sim 530 \mathrm{fs}$ FWHM in the resulting $640 \mathrm{Gbit} / \mathrm{s}$ data signal. An eye diagram of the $640 \mathrm{Gbit} / \mathrm{s}$ data is also shown in Fig. 1. This shows clear and open and well equalised $640 \mathrm{Gbit} / \mathrm{s}$ data eyes. The flat-top pulses are derived from an identical pulse source and pulse compressor followed by a 13-nm band-pass filter, yielding $500 \mathrm{fs}$ input pulses to the LPG. A variable time delay is put in the LPG arm to align the FT-pulses with the data pulses, and a 


\section{OWS5.pdf}

polarisation controller is put in front of the LPG to characterise its polarisation dependence. A $0.5 \mathrm{~m}$ dispersion compensating fibre is put after the LPG to trim the dispersion in the path to the demultiplexer. The FT-pulse acts as control pulse in a non-linear optical loop mirror (NOLM) including $50 \mathrm{~m}$ HNLF. All the HNLF is kindly provided by OFS Fitel Denmark and have a $\gamma \sim 10 \mathrm{~W}^{-1} \mathrm{~km}^{-1}$, dispersion slope $\sim 0.018 \mathrm{ps} / \mathrm{nm}^{2} \mathrm{~km}$, zero dispersion at $1551 \mathrm{~nm}$ for the NOLM-HNLF, and $-1.2 \mathrm{ps} / \mathrm{nm} / \mathrm{km}$ dispersion at $1550 \mathrm{~nm}$ with a slope of $0.003 \mathrm{ps} / \mathrm{nm}^{2} \mathrm{~km}$ for the compression HNLFs. The control and data wavelengths are aimed at being roughly symmetrically placed around the zero dispersion wavelength in the NOLM to minimise walk-off, so the data is at $1560 \mathrm{~nm}$ and the FT control is at $1542 \mathrm{~nm}$. The demultiplexing quality is evaluated in terms of bit error rates with the receiver power measured after the NOLM at the input to the $10 \mathrm{Gbit} / \mathrm{s}$ pre-amplified receiver.

\section{Flat-top pulse generation and characterisation}

The LPG has a linear transfer function of $\mathrm{j}\left(\omega-\omega_{0}\right)$, essentially differentiating the electric field [5]. Spectrally offsetting the input creates a superposition of the original pulse shape with its differential, which consists of a temporal double-pulse. The original pulse can fill the gap in the double-pulse. For a certain detuning, the gap disappears completely, forming a perfect FT waveform. The transfer function corresponds to a spectral dip in terms
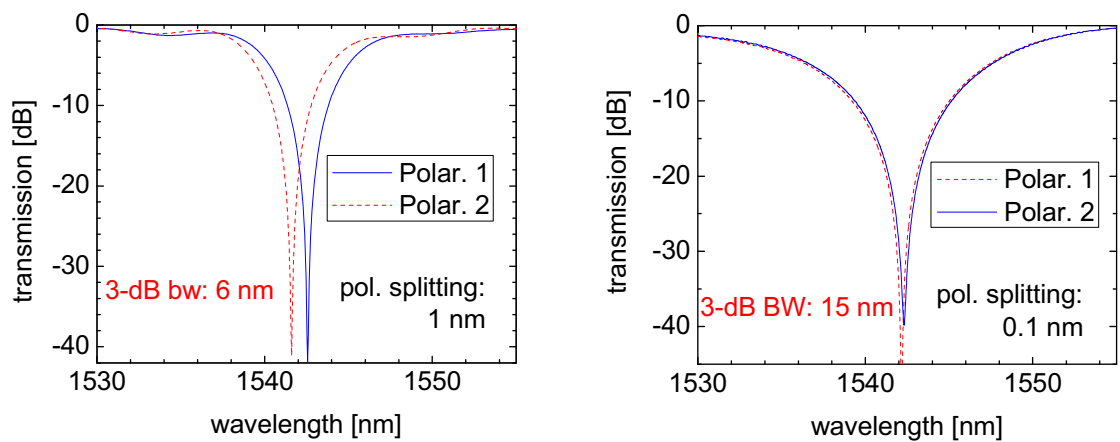

Figure 2.Bandwidth and polarisation. Left: LPG used in [6] with $6 \mathrm{~nm}$ width and a $1 \mathrm{~nm}$ polarisation splitting. Right: New LPG for this work with $15 \mathrm{~nm}$ bandwidth and only 100 pm polarisation splitting.

of intensity. Fig. 2 shows these characteristic dips for the LPGs used in [6] and in this work. The bandwidth is increased to $15 \mathrm{~nm}$ and the polarisation dependence is reduced to only $0.1 \mathrm{~nm}$, which is negligibly small for the spectral widths considered here. The LPGs are routinely made in a side-exposure of an optical fiber, which yields non-uniform refractive index change across the fiber cross-section, giving a slight birefringence. This causes the resonance frequency to vary depending on the polarisation of the input light, and results in a $1 \mathrm{~nm}$ polarisation splitting in the LPG used in [6]. For this paper, an inscription technique that illuminates the fiber from three sides at the same time is implemented [7]. This greatly reduces the photoinduced birefringence. Also the $15 \mathrm{~nm}$ bandwidth of the LPG covers the entire pulse spectrum, which ensures good flat-top pulse generation.
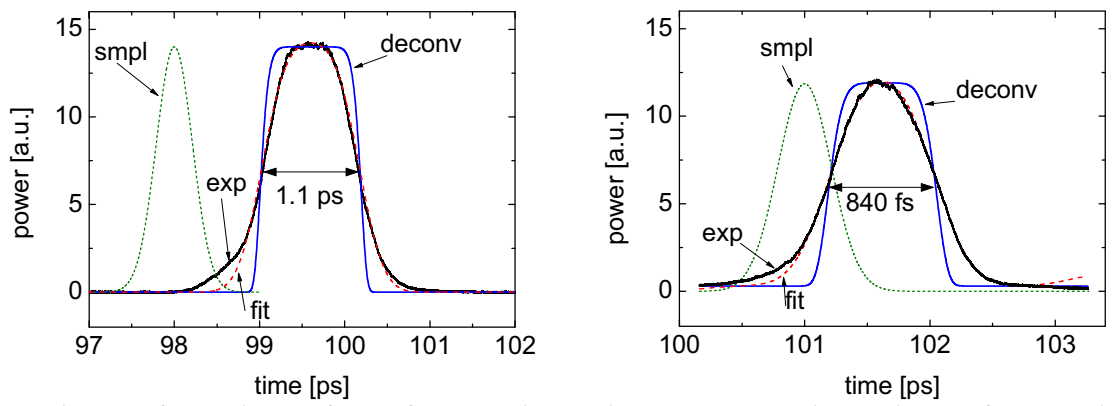

Figure 3. Cross-correlations of FT-pulses. Left: $700 f_{s}$ input pulse results in 1.1 ps FT-pulse. Right: $500 f_{s}$ input pulse results in $840 f$ s FTpulse. The deconvoluted FT-pulses are super-gaussian of order $m=3$, and the width can be tuned by the input FWHM.

Fig. 3 shows the temporal output of the LPG in two cases. Fig 3 (left) is for a gaussian-shaped 700 fs FWHM input pulse, resulting in a 1.1 ps flat-top output pulse. Fig. 3 (right) is for a $500 \mathrm{fs}$ input pulse, giving an $840 \mathrm{fs}$ FT-pulse. The figure shows several traces: One is the measured cross-correlation ("exp"), one is the sampling pulse ("smpl"), one is the true FT-pulse ("deconv") when deconvoluting the sampling pulse out of the "exp"-trace and the last is the fitted curve ("fit") to the "exp"-trace, i.e. the convolution of the "smpl" and the "deconv" traces. There is very good 


\section{OWS5.pdf}

agreement between the measured and the fit traces.The deconvoluted FT-pulses are in both cases super-gaussian of order $m=3$. To characterise the quality of the generated pulses further, BER mesurements are performed.

\section{BER results}

Fig. 4 (right) shows an eye diagram of all 64 OTDM channels, showing a high quality signal with all channels well equalised and with the appropriate separation, i.e. no channels are overlapping or disturbing each other. Fig. 4 left) shows the demultiplexed BER performance when using the $840 \mathrm{fs}$ FT-pulse and a $700 \mathrm{fs}$ gaussian pulse (made by detuning the LPG-dip away from the pulse spectrum), as they have the same 1/e-width. The demultiplexing is errorfree with less than $0.5 \mathrm{~dB}$ penalty to the $10 \mathrm{Gbit} / \mathrm{s}$ back-to-back in the FT-case. Using the gaussian results in a 0.7 $\mathrm{dB}$ additional penalty, probably due to timing jitter. Note that the same channel is demultiplexed in both cases, which is ensured by detuning the LPG, whilst monitoring the demultiplexing.
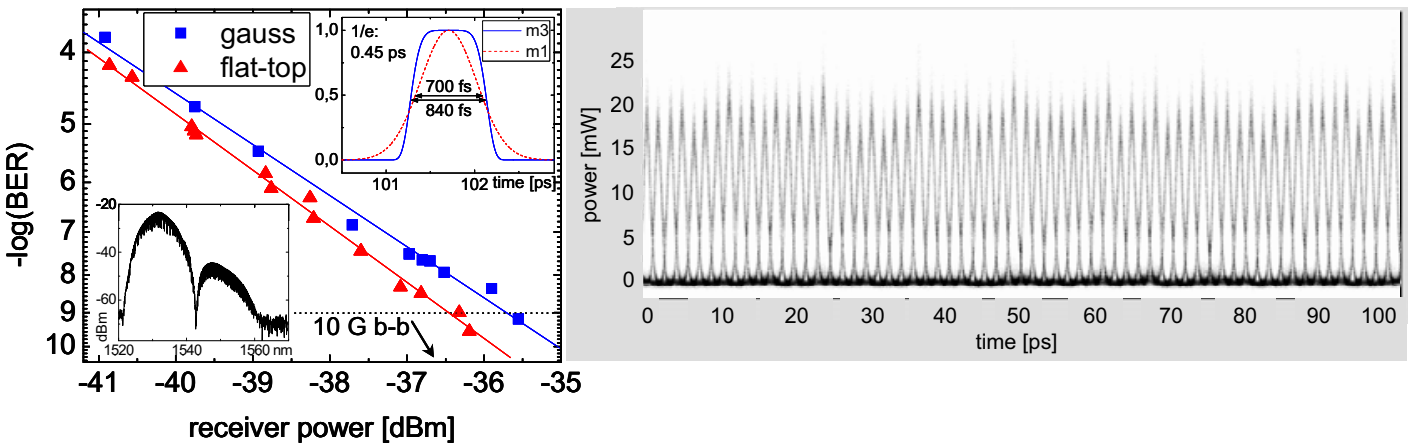

Figure 4. Left: BER performance when using FT or gauss pulse on the same channel. Insets show the super-gaussian and the gaussian control pulses, and also the spectrum of the $840 \mathrm{fs}$ FT-pulse. Right: $640 \mathrm{Gbit} / \mathrm{s}$ eye diagram.

Fig. 5 shows the measured timing tolerance BER curves, as measured when displacing the control pulse with respect to the data pulses. At $5 \mathrm{~dB}$ receiver power above the receiver sensitivity the gauss has a $110 \mathrm{fs}$ error-free
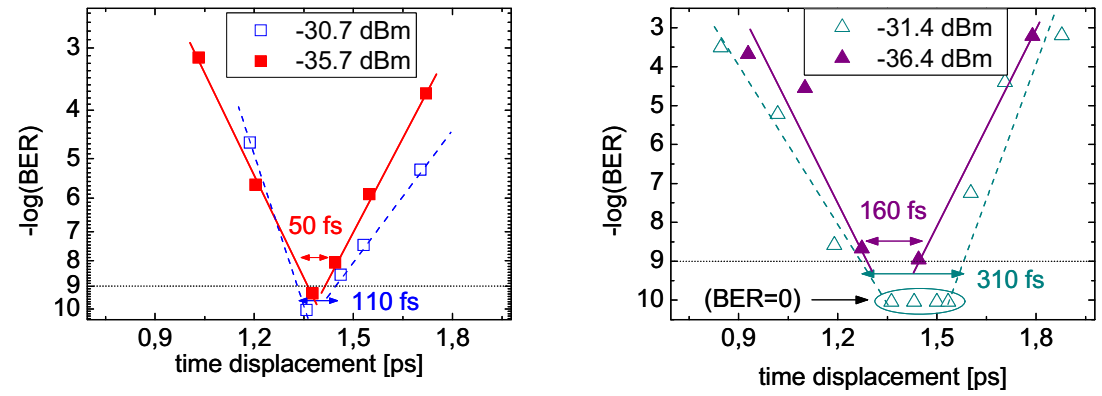

Figure 5. Timing tolerance at $640 \mathrm{Gbit} / \mathrm{s}$, measured at the receiver sensitivity and at $5 \mathrm{~dB}$ more power to the receiver. Left: $700 \mathrm{fs}$ gaussian control pulse. Right: $840 \mathrm{fs}$ FT control pulse giving up to $310 \mathrm{fs}$ tolerance with $B E R=0$.

timing tolerance, where the FT has a $310 \mathrm{fs}$ tolerance, where the absolute BER is zero. This corresponds to the flat part of the FT pulse and is about $20 \%$ of the time slot, and almost three times greater than for the gaussian.

\section{Conclusion}

We have reported on a polarisation-independent long-period fiber grating used to create sub-picosecond flat-top pulses. An $840 \mathrm{fs}$ pulse was created and for the first time used for $640 \mathrm{Gbit} / \mathrm{s}$ demultiplexing with $<0.5 \mathrm{~dB}$ penalty to the back-to-back and with an error-free timing tolerance of $310 \mathrm{fs}$, i.e. $20 \%$ of the time slot. These findings clearly demonstrate the potential of the LPG-generated flat-top pulses.

\section{References}

[1] H.G. Weber et al, "Single channel 1.28 Tbit/s and 2.56 Tbit/s DQPSK transmission," Electron.Lett., Vol.42, Issue 3, (2006), pp 178-179

[2] S. Watanabe et al, " $160 \mathrm{Gbit} / \mathrm{s}$ optical 3R-regenerator in a fiber transmission experiment", OFC 2003, post-deadline paper PD 16-1

[3] F. Parmigiani et al, J. Lightwave Technol., Vol. 24, Issue 1, (2006) pp 357-364

[4] L.K. Oxenløwe et al, In proceedings of ECOC' 06, Cannes, France, September 2006. Paper We2.3.4

[5] Y. Park et al, Opt. Express, Vol. 14, Issue 26, (2006), pp 12670-12678

[6] L.K. Oxenløwe et al, J. Select. Topics in Quantum. Electron, Vol. 14, No. 3 (2008), pp 566-572

[7] V. Grubsky et al, Photon. Technol. Lett., Vol. 18, No. 21, pp. 2296-2299, 2006. 